\title{
Être (d'accord) ou ne pas être (d'accord) ? L'enjeu du débat Aubry-Hollande : entre indépendance et unité
}

\author{
Arnaud Richard \\ Laboratoire Praxiling UMR 5267 - Université Montpellier 3 - CNRS \\ arnaud.richard@gmail.com \\ Marion Sandré \\ Laboratoire Praxiling UMR 5267 - Université Montpellier 3 - CNRS \\ marion.sandre@gmail.com
}

\section{Introduction}

Le débat de l'entre-deux tours des primaires socialistes, qui a opposé Martine Aubry (désormais MA) et François Hollande (désormais FH) le 12 octobre 2011, est inédit à la télévision française. Cette interaction présente des caractéristiques génériques particulières : s'il s'agit bien d'un échange relevant du genre débat politique télévisé (Sandré, 2010 : 160-171) - et plus précisément appartenant à la famille des «débats de l'entre-deux tours »-, il est aussi un débat interne à un parti politique visant à élire le candidat de cette formation pour les prochaines élections présidentielles françaises, en 2012. Ce débat doit être défini à partir de ces deux critères. C'est un débat politique télévisé en ce qu'il s'agit d'une interaction médiatisée, présentant un échange entre deux personnalités politiques. Il est organisé par l'animateur représentant la chaine de diffusion (France 2) David Pujadas (désormais DP), et ponctuellement par trois journalistes : Fabien Namias (désormais FN) - responsable du service politique de France 2-, Patrick Cohen (désormais PC) - journaliste à la radio France Inter - et Françoise Fressoz (désormais FF) - éditorialiste au journal Le Monde. À cet échange en présence s'ajoute une scène englobante construite entre les interactants sur le plateau de télévision et les téléspectateurs. Le fait que ce soit un face à face entre les deux candidats arrivés en tête des votes à l'issue du premier tour des élections renforce l'antagonisme du genre débat: les deux se battent l'un contre l'autre et doivent gagner l'adhésion des téléspectateurs-électeurs, i.e. leur vote le dimanche suivant pour arriver en tête du second tour. L'objectif est donc d'opposer les deux candidatures et pour MA comme pour FH de prouver qu'il est meilleur que l'autre et/ou que l'autre est moins bon. C'est aussi un débat interne à une formation politique : en ce sens, les deux candidats partagent les mêmes valeurs politiques, ont un projet commun (le projet voté par les socialistes) et s'opposent tous les deux à la candidature - non encore officielle - du candidat de la formation politique adverse. L'objectif d'une telle élection est donc de montrer, au-delà des divergences personnelles, une cohésion de parti et de partager les mêmes idées contre un adversaire externe au débat. On peut donc caractériser cette interaction par la nécessité à la fois de montrer le dissensus entre les deux candidats - et proposer ainsi un vrai débat - et de construire un consensus plus global - afin de donner une image unie du parti socialiste. Le rôle des animateurs d'ailleurs est bien celui de l'arbitre entre ces deux tensions : ils interviennent fréquemment pour préciser les points de divergence et les points de convergence entre les deux candidats. Cet article se propose d'analyser ces deux caractéristiques inhérentes à ce nouveau sous-genre qu'est le face-à-face interne à une formation politique, et de montrer comment les deux candidats se positionnent l'un par rapport à l'autre.

Après avoir analysé d'autres débats télévisés ${ }^{1}$ et montré que les marques d'accord étaient très peu développées dans le face à face politique contrairement au débat culturel, l'étude des phénomènes 
consensuels et dissensuels dans ce nouveau type de débat télévisé permettra d'en proposer une approche définitoire en relation avec ces autres débats. Ce travail s'inscrit dans le champ de l'analyse du discours, telle qu'elle est développée aujourd'hui en France (par exemple Dufour et Rosier, 2012; Guilbert, 2011 ; Hailon et al. (à paraître) ; Richard, 2007 ; ou encore Charaudeau, 2005), en s'ouvrant à des courants connexes: nous utiliserons ainsi différents concepts issus des théories énonciatives, des travaux sur le dialogisme et de l'analyse interactionnelle (notamment Bres et al., 2005 ; Rémi-Giraud et Constantin de Chanay, 2007 ; ou Vérine et Détrie, 2011), permettant d'étudier les différents éléments discursifs, conversationnels ou encore non verbaux témoignant l'accord ou le désaccord.

Avant d'être des adversaires, MA et FH sont des partenaires politiques : nous proposerons donc un plan en deux temps. On analysera d'abord les différentes occurrences montrant le consensus entre les deux candidats, puis celles marquant le dissensus. On pourra ainsi comparer les différentes formes que prend la formulation de l'accord et du désaccord et les différentes utilisations qu'en fait chacun des adversaires.

\section{Manifester son accord}

Ce débat n'est pas un débat politique télévisé ordinaire, nous l'avons dit. En effet, les deux participants qui se retrouvent en face à face ne sont pas des adversaires mais bien des alliés politiques. Ainsi ils se connaissent personnellement, s'appellent mutuellement par leurs prénoms, se tutoient et répondent à la première question de DP en assurant chacun à l'autre son amitié. Il leur arrive de rire ensemble de leurs bons mots : le ton donné est d'emblée amical et collaboratif. Cependant les deux finalistes doivent avant tout défendre leur propre candidature. La manifestation d'un consensus peut alors être plus ou moins appuyée. On traitera les différents cas selon un axe progressif : d'abord lorsqu'un candidat manifeste simplement son accord avec le discours de l'autre, puis lorsqu'il le valide plus franchement, enfin lorsqu'il enchaîne sur la parole de l'autre, adoptant ainsi clairement le même point de vue.

\subsection{S'accorder au discours de l'autre}

L'accord peut être exprimé de façon plus ou moins claire : on analysera ainsi les cas où l'accord est formulé sous forme d'adverbe confirmatif et ceux où il est formulé sous forme de répétition textuelle.

\subsubsection{Accord minimal}

La formulation d'un accord minimal se fait sous forme d'adverbe d'acquiescement, dont l'utilisation permet à un locuteur de montrer son accord de façon ponctuelle, sans prendre véritablement la parole à l'autre. En analyse interactionnelle, ces adverbes d'affirmation sont souvent considérés comme des régulateurs, i.e. des unités interactives émises par l'auditeur d'un message pour montrer son investissement dans l'échange ${ }^{2}$. On différencie habituellement les régulateurs, selon la fonction qu'ils remplissent dans l'interaction. Nous ne retiendrons ici que les signaux qui manifestent l'accord de l'interlocuteur par rapport aux propos du locuteur en place, c'est-à-dire les continueurs : signaux par lesquels «le récepteur évalue positivement l'énoncé du locuteur» (De Gaulmyn, 1987: 207). On retrouve ainsi plusieurs occurrences de «mmh », «(et) oui », «bien sûr », principalement du fait de $\mathrm{MA}^{3}$. Il arrive que ces régulateurs soient émis en chevauchement, comme dans l'exemple 1 :

$(1)^{4}$

213a FH [tout tout l'monde] défend les emplois d'avenir $\uparrow$ mais faut-il encore qu'on puisse les les renouveler c'est-à-dire les mettre pour moi dans les QUARtiers où il y a \&

bien sûr 
213b FH \& le plus de chômage [où les jeunes sont à quarante pour cent d'chômage (hochement de tête de MA) + où on a besoin d'un soutien scolaire où on a besoin d'accompagnement $\downarrow$ ]

C'est MA, en 214, l'auteur du régulateur sous forme de la locution adverbiale « bien sûr ». Sa prise de parole est prononcée intégralement en chevauchement alors que FH conserve la parole et poursuit son tour de parole 213. Il s'agit ici pour MA simplement de formuler son accord avec la nouvelle idée introduite par FH (mettre les emplois d'avenir dans les quartiers). Elle indique de cette façon qu'elle soutient la proposition de son adversaire, sans l'interrompre ou développer elle-même cette idée. Son hochement de tête ensuite répond exactement à la même stratégie : ce geste indique clairement l'approbation. Il est fréquemment utilisé par les participants à un débat pour montrer son accord sans intervenir discursivement dans l'échange (cf. Sandre, 2011a : 246). Il faut toutefois noter que la plupart de ces manifestations non verbales échappent à l'analyse, le réalisateur de l'émission télévisée choisissant bien souvent de filmer le locuteur en place plutôt que l'interlocuteur.

Ces phénomènes de régulation correspondent à la manifestation verbale minimale de l'accord. Dans ce cas, l'auteur de la reprise se contente de marquer ponctuellement son accord sans le commenter. Cela se justifie par la forme que prennent ces reprises : les régulateurs ne sont pas des tours de parole au sens strict de l'analyse interactionnelle. Leur auteur ne prend pas véritablement la parole, ni ne développe son point de vue, mais valide simplement celui du locuteur en place.

\subsubsection{Accord montré}

L'accord peut être montré sous forme de reprise textuelle ${ }^{5}$ : en répétant les mêmes mots, le locuteur signale qu'il partage le même avis. Cette répétition peut être marquée par le locuteur qui l'utilise, ou par l'interlocuteur qui l'identifie. Les exemples 2 et 3 présentent ces deux cas :

(2)

228 FH $[\ldots]$ c'est une BELLE idée qu'un senior puisse être utile et puisse partir en retraite avec ses droits de plein de plein exercice voilà pourquoi je pense bien sûr (main droite en direction de $M A$ ) qu'on négociera avec les partenaires sociaux parce que je pense qu'il n’y a pas de négociations si nous n'sommes pas conscients que la démocratie sociale est un atout pour notre pays $[\ldots]$

(3)

280a FH la réforme fiscale c'est la réforme qui doit être globale $\downarrow$ l'impôt sur le revenu qui doit être fusionné avec la c s $\mathbf{g}^{6} \downarrow$ ça c'est l'impôt sur le \&

281 MA ça c'est un premier point

280b FH \& l'ensemble des revenus [travail comme capital avec le même barème on aura réglé un premier problème $\downarrow]$

Dans l'exemple 2, FH fait référence au discours de MA, qui proposait précédemment de «négocier [avec] tous les syndicats » (221). Le candidat reprend le même verbe et élargit la portée de la négociation à tous les " partenaires sociaux ». Cette reprise du discours de son adversaire est clairement indiquée par l'auteur : il utilise la locution adverbiale «bien sûr » en réponse à son discours et montre son interlocutrice de la main. Ce geste d'ouverture illustre la rencontre d'idées entre les deux candidats ${ }^{7}$ (Calbris, 1979: 91). Il inscrit donc lui-même MA dans son discours pour indiquer qu'il lui reprend l'idée de la négociation.

C'est encore FH l'auteur de la répétition dans l'exemple 3 : il propose de fusionner l'impôt sur le revenu avec la CSG, reprenant ainsi les mots de MA « on relie l'impôt sur le revenu et la CSG » (246). 
Il ne fait pas référence au discours de son adversaire, comme précédemment. C'est donc elle qui intervient en 281 pour indiquer qu'il s'agit d'un consensus dans leur projet commun de réforme fiscale. La candidate se positionne alors clairement dans la même lignée que son interlocuteur. Il faut noter cependant que sa prise de parole reste en chevauchement et que FH conserve la parole et poursuit sa démonstration sans prendre en compte l'intervention de son adversaire dans son propre discours.

Les phénomènes de répétition correspondent à une manifestation montrée de l'accord. Toutefois, sans l'intervention de l'auteur du discours cité ou de celui du discours citant, ces occurrences sont plus difficiles à identifier (il faut alors analyser précisément le contenu du discours pour repérer les reprises). Nous voulions ici montrer les cas où l'accord est délibérément montré par l'un ou l'autre locuteur engagé dans l'échange.

Formuler un accord peut se faire sous forme plus ou moins explicite, nous avons vu ici l'expression minimale - sous forme de régulateurs - et la simple répétition du discours de l'autre (qui peut être clairement identifié par le locuteur citant ou le locuteur cité).

\subsection{Valider le discours de l'autre}

Pour témoigner d'un accord, il est possible de valider le discours de l'autre. Le candidat peut ainsi se positionner personnellement comme étant du même avis, ou plus largement indiquer qu'il s'agit d'un consensus global au sein du parti socialiste.

\subsubsection{Validation personnelle}

Le consensus entre les candidats peut être exprimé de façon plus explicite que précédemment. Ainsi, il n'est pas rare qu'un des candidats formule clairement son accord. On retrouve les expressions : «ça on est d'accord " (MA 457), «je suis là-dessus totalement d'accord» (MA 272) et «nous sommes d'accord là-dessus » (FH 172). Il arrive aussi que l'animateur du débat demande à l'un ou à l'autre s'il est d'accord avec son adversaire, une réponse affirmative correspondant alors à un accord explicite, comme c'est le cas dans l'exemple suivant :

(4)

290 FH [...] vous savez la seule question qu'il faut se poser + par rapport à deux mille douze c'est savoir si il y aura des augmentations de prélèvement $\downarrow+$ hélas il y en aura $\downarrow$

291 MA bien sûr

293 DP [là-dessus vous êtes d'accord $\uparrow]$

295 MA [bien sûr]

Dans ce passage, MA exprime un accord minimal avec le discours tenu par FH en 291 sous forme de régulateur. DP intervient alors pour lui demander de confirmer son intervention : la réponse de MA en 295 correspond donc à un accord explicite. L'affirmation du consensus est demandée par l'animateur, qui remplit ici pleinement sa fonction d'arbitre : son rôle interactionnel est de révéler les positions de chacun des candidats et de mettre en valeur les points d'accord et de désaccord entre les deux. Il soulève ici un point particulièrement important : les augmentations de prélèvement sont particulièrement non électoralistes, il est donc important, dans le cadre d'une élection, de savoir si les deux candidats sont d'accord sur ce sujet. L'intervention de MA permet de montrer un parti uni autour des mêmes préoccupations, ce qui est encore plus clair dans les exemples suivants. 


\subsubsection{Validation collégiale}

Ici, il ne s'agit plus seulement d'un accord ponctuel entre les deux candidats, mais d'annoncer un accord au sein de tout le parti socialiste. Le candidat ne parle plus alors en son nom propre mais au nom de la formation politique et utilise les pronoms personnels nous et on. On retrouve des expressions comme «nous l'avions dit en deux mille huit tous ensemble» (MA 310), «l'engagement que nous prenons enfin je veux pas me différencier là-dessus » (FH 615), « maintenant au parti socialiste tout le monde est d'accord » (MA 617), « là je crois qu'on est tous d'accord » (MA 623). Le corpus présente également un exemple où un accord explicite vient entériner un accord montré :

(5)

627 FH deuxième réforme à faire $\uparrow$ il n'a: pas à faire pression sur la justice $\downarrow$ il y aura donc euh l'indépendance de la magistrature et euh la fin euh de la nomination des parquets 'fin des procureurs comme c'est le cas aujourd'hui $\downarrow$ il faut que ce soit une république EXemplaire également + sur le plan euh + des nominations $\downarrow$ le CONseil constitutionnel $\downarrow+$ moi je vais faire euh la proposition d'une réforme du conseil constitutionnel $\downarrow+$ c'est plus possible que ça se fasse + avec des nominations qui viennent du président $>$ d'la république $<+$ ou des présidents de chacune des assemblées il faudra que çe soit CONtrôlé par le parlement parce que c'est + finalement une façon des personnalités nouvelles à des décisions très importantes $h$ et puis république exem\&

628 MA je crois qu'on est tous d'accord c'est dans notre projet il faut VRAIment le faire $\downarrow$ $629 \mathrm{FH} \quad$ \&plaire également sur la question de la réforme des modes de scrutin [...]

Les candidats parlent ici des contre-pouvoirs par rapport au président de la République. FH, à la suite de $\mathrm{MA}$, énumère les réformes qu'il veut mener et insiste alors sur l'indépendance de la magistrature, reprenant quasiment mot pour mot le discours de MA : « il faut redonner une indépendance à la justice en modifiant le conseil supérieur de la magistrature pour le rendre indépendant $\downarrow$ la nomination + la promotion des procureurs des magistrats doit être totalement indépendante du pouvoir politique » (624). Il s'agit donc ici d'un accord montré. Puis FH va plus loin et enchaîne sur la réforme du conseil constitutionnel, dont MA n'a pas parlé. Cette dernière intervient, en 628, pour indiquer qu'il s'agit d'un accord global au sein du parti (elle utilise le pronom on, renforcé par le pronom tous et parle de «notre projet ») et même d'une nécessité politique au-delà des clivages (comme l'indique la fin de son énoncé, avec le verbe falloir et l'adverbe vraiment, dont elle accentue la première syllabe). Cette prise de parole, énoncée intégralement en chevauchement pendant que FH conserve la parole, vient expliciter l'accord et indiquer qu'il s'agit d'un consensus général.

Dans l'exemple 6, l'accord entre les candidats fait l'objet d'une négociation autour de la prise de parole :

(6)

238 FN [...] la fiscalité c'est un leVIER pour retrouver de la marge de manœuvre $+\mathrm{h}$ on a vu aujourd'hui la majorité s'apprête à appliquer une TAXE éceptionnelle exceptionnelle + sur les plus haut revenus $\uparrow$ si l'un de vous deux $+>$ est élu président de la république $<$ est-ce que + l'une de vos premiers mesures + sera + un NOUvel impôt $\downarrow$ une NOUvelle taxe $\downarrow$ sur les revenus les plus élevés aller + plus loin que c'qui est proposé aujourd'hui

239 FH ben su- (main droite en direction de MA)

240 MA alors françois euh pardon (main gauche en direction de FH)

241 FH pardon euh

242a MA 'fin $\mathrm{j}$ 'vais aller dire une chose pour dire \&

$243 \quad$ FN $\quad$ vous répondrez tout les deux $\downarrow$

242b MA \& qu'on était d'accord 
244 DP allez-y martine aubry vous avez encore un léger temps de retard

245 FH si martine aubry veut dire qu'elle est d'accord je lui laisse la parole (sourire)

246 MA ben euh je nous avons voté tous ensemble à l'unanimité un projet que nous avons préparé tous ensemble $\downarrow$ avec nos experts $\downarrow[\ldots]$

Suite à la question de FN, les deux candidats commencent à parler en même temps, produisant ainsi un « chevauchement en début de tour » (Sandré, 2010) en 239 et 240. Ils s'excusent ensuite mutuellement jusqu'à ce que $\mathrm{FH}$, en 245 , cède la parole à MA après qu'elle l'a assuré de présenter un point de vue commun (242). Si elle commence sa prise de parole 246 par le pronom personnel je, elle le remplace immédiatement par le pronom nous, renforcé par la locution «tous ensemble » (répétée deux fois). Il s'agit ici explicitement de défendre l'unanimité au sein du parti socialiste. L'intervention de FH en 245 est révélatrice de l'enjeu que sont les passations de parole dans le débat pour affirmer son avis. La cession de la parole est ici liée à la promesse de proposer un point de vue convergent. En 239 et 240, on retrouve le même geste que dans l'exemple 2, mais il est ici effectué par les deux candidats simultanément, illustrant ainsi gestuellement cette rencontre d'idées (particulièrement saillante grâce à une prise de vue globale derrière les deux débatteurs).

Valider le discours de l'autre revient à exprimer explicitement un accord. Nous avons vu plusieurs formes de validation : il peut s'agir d'un accord seulement entre les deux candidats ou d'un accord plus général au sein de la formation politique. La validation peut ainsi être de portée personnelle ou collégiale.

\subsection{Enchaîner sur le discours de l'autre}

L'accord peut être exprimé de façon plus inattendue sous forme d'enchaînement sur le discours de l'autre: en prolongeant ainsi la parole de son interlocuteur, on lui prête sa voix et on adopte délibérément la même position. Tous les cas relevés sont des enchaînements dialogiques interlocutifs (Sandré, 2010 et 2011b), c'est-à-dire se réalisent comme inclusion de paroles dialogiques dans une paire adjacente (Sacks et al., 1974). L'auteur de l'enchaînement produit une intervention à la suite du locuteur précédent en l'adaptant déictiquement à lui et en relation avec son discours : l'enchaînement dialogal est présenté par le second locuteur comme un enchaînement intradiscursif. De cette façon, il s'immisce dans le discours de l'autre et parle à sa place, pour poursuivre son idée ou défendre son point de vue.

L'enchaînement peut se produire à différents moments de la prise de parole du locuteur précédent : soit à la fin du tour (à une place de transition pertinente), soit au milieu du tour, sous forme de chevauchement ou d'interruption. Dans l'exemple 7, MA intervient à la fin du tour de FH :

(7)

$428 \mathrm{FH} \quad[\ldots]$ je pense qu'il faut + laisser cette règle d'un service minimum $\uparrow$ mais ça doit être défini par les partenaires sociaux et non pas par la loi comme ça a été fait $\downarrow$

429a MA ça a d'ailleurs été \&

430 DP martine aubry

429b MA \& fait dans certaines entreprises a la $\mathbf{r}$ a $\mathbf{t} \mathbf{p}^{9}$

431 DP à la $\mathrm{r}$ a t $\mathrm{p}$ notamment

$432 \mathrm{Ma}$ et euh je pense que ça marche mieux que quand on l'impose hein euh on le voit euh à tout moment $\downarrow$

Les candidats sont ici interrogés sur le service minimum garanti en cas de grève (instauré par le gouvernement au pouvoir), FH commence à répondre et MA, en 429, prend la parole dès qu'il a terminé (avant même qu'on lui donne la parole, comme c'est fait en 430) pour poursuivre et exemplifier l'idée 
de FH. Son intervention pourrait tout à fait être énoncée par le candidat: elle reprend la même construction syntaxique (le pronom démonstratif ça sujet et le verbe faire au passif) et indique qu'il s'agit d'un ajout avec la locution adverbiale d'ailleurs. On peut noter que la suite de son discours, en 432, ne relève plus du même procédé puisqu'elle se positionne dès le départ comme énonciatrice (en utilisant le pronom je) et peut ensuite, puisqu'on lui a donné officiellement la parole, développer son avis - très proche au demeurant de celui de FH.

Tous les autres exemples relevés sont des cas d'intervention en milieu de tour: l'auteur de l'enchaînement dialogique prend la parole alors que son interlocuteur n'a pas achevé son discours. S'il s'agit bien d'un « raté du système des tours » (Kerbrat-Orecchioni, 1990 : 172) sur le plan du partage de la parole, sur le plan relationnel, ce phénomène correspond nettement à une visée collaborative.

Le corpus présente un certain nombre de cas où l'intervention du locuteur se fait sous forme de chevauchement: il ne prend pas la parole à l'autre, mais parle en même temps que lui pour lui témoigner son soutien. S'il arrive que les deux locuteurs disent la même chose en même temps ${ }^{10}$, le plus souvent l'énoncé en chevauchement est ensuite repris par le premier locuteur, comme dans l'exemple suivant $^{11}$ :

(8)

159a FH $\quad[.$.$] moi je propose que la réforme fiscale soit débattue dès notre arrivée à la$ responsabilité du pays $\downarrow+$ et deuxièmement il va falloir soutenir la croissance $\downarrow$ de ce point de vue là martine a raison mais les effets de cette politique n'arriveront qu'en deux mille treize ou deux mille quatorze + alors nous verrons où nous en serons en deux mille treize il faudrait que nous arrivions à à avoir ré\&

$160 \quad$ MA trois pour cent

159b FH \&duit nos déficits à trois pour cent $\downarrow$ (main droite en direction de MA) de ce point de vue + nous avons fixé l'objectif ensemble [...]

Le phénomène qui nous intéresse se trouve en 160 : MA intervient dans le discours de FH et produit une coénonciation ${ }^{12}$. Elle propose une suite «trois pour cent» à l'énoncé de FH « il faudrait que nous arrivions à ». Ce dernier, en même temps, poursuit sa propre programmation syntaxique et complète par une proposition subordonnée infinitive " avoir réduit nos déficits à trois pour cent». Le pourcentage, cité par MA, était bien l'aboutissement de son propos, comme l'indique son geste en 159b : il la désigne comme il le faisait dans l'exemple 2, afin de montrer la rencontre de leur idée. Il faut par ailleurs noter que cet accord a été introduit par FH précédemment : en 159a, il valide le discours de MA « de ce point de vue là martine a raison », et il clôture cette intervention en $159 \mathrm{~b}$ en précisant qu'ils ont "fixé l'objectif ensemble ». Le consensus entre les deux candidats est donc ici clairement établi par $\mathrm{FH}$, en cela l'intervention de MA en 160 est tout à fait compréhensible. Elle se sent investie dans le discours de son interlocuteur et montre ainsi qu'elle l'approuve. Cet exemple propose donc plusieurs phénomènes témoignant l'accord entre les deux candidats.

Le corpus présente aussi des cas où le témoignage de l'accord ne prend plus la forme d'un énoncé en chevauchement mais d'un énoncé interruptif, toujours à visée collaborative. Le corpus présente ainsi deux cas de soutien linguistique (Sandré, 2010) en 259 et 523, où MA interrompt FH après que ce dernier a marqué une hésitation pour l'aider lexicalement. Le candidat reprend alors la parole immédiatement pour entériner la proposition de son interlocutrice (524) ou pour reprendre le contenu avec ses propres mots (260) : il valide de cette façon les interventions de MA, pertinentes sur le plan interactionnel (elle lui vient en aide pour la construction de son discours) et relationnel (elle montre ainsi qu'elle est tout à fait d'accord avec ses propositions).

Le dernier exemple, le seul enchaînement du fait de FH, est un peu plus complexe : 
(9)

417 MA [...] est-ce que pour les français le service est de meilleur qualité et moins couteux quand c'est fait par le public $\uparrow \mathrm{h}$ ou est-ce qu'il vaut mieux qu'ils payent directement $\downarrow+$ quand je r'garde ce qui se passe aux états-unis ou en grande bretagne $\downarrow$ où on PAYE les études $\downarrow+$ où on PAYE euh pour se faire soigner $\downarrow$ alors oui $\uparrow$ ils ont beaucoup moins + effectivement de fonctionnaires et d'agents publiques \

418 PC mais quand vous r'gardez l'Allemagne

419 MA mais ça leur coute ++ mais ça leur coute beaucoup plus cher $+>$ ça leur coute beaucoup $<$ oui mais en Allemagne $\backslash$

420 FH mais en Allemagne c'est un pouvoir fédéral où il y a de grandes régions $\downarrow$

421 MA c'est la régionalisation et c'est la région qui paye $\downarrow[\ldots$

L'énoncé interruptif de FH, en 420, n'apporte pas un soutien lexical mais un argument pour aider la candidate à répondre à la question de $\mathrm{PC}$ en 418 . Pour défendre le modèle français, MA le compare aux modèles anglos-saxons (417), l'animateur l'interrompt alors pour lui opposer un autre modèle: l'Allemagne. Dans un premier temps, MA ne répond pas et poursuit son énoncé interrompu, même si l'intervention de PC la déstabilise quelque peu comme l'indique les répétitions dans son discours (419). Elle modifie alors sa thématique discursive au milieu de son énoncé pour répondre à l'animateur. En même temps, FH intervient pour répondre lui aussi à PC en avançant l'argument de la régionalisation, ensuite repris par MA en 421. L'interruption avec chevauchement du candidat joue donc ici clairement le rôle d'adjuvant aux côtés de son opposant dans le débat face à l'animateur. Il ne s'agit plus seulement d'un accord entre les deux, mais d'une réelle coalition : ils mettent leurs arguments en commun pour défendre leur vision du service public français.

Tous ces cas de coénonciation montrent, outre un accord entre les deux candidats (ils pensent la même chose, défendent les mêmes arguments et partagent souvent les mêmes mots), une vraie collaboration discursive qui les amènent à parler de concert. Il faut noter que la grande majorité des exemples sont le fait de MA.

Les phénomènes discursifs témoignant un accord entre les candidats sont nombreux et variés. Le consensus peut être plus ou moins appuyé, plus ou moins explicite, la collaboration entre les deux peut apparaître de façon plus ou moins claire. Nous avons présenté les différentes occurrences depuis les formes minimales jusqu'aux preuves plus accentuées. Ces multiples manifestations d'accord entre les deux sont inhabituelles dans un face à face politique. Il est intéressant de noter que habituellement, les marques d'accord sont beaucoup plus rares, et se cantonnent au discours énoncé à l'intérieur du débat (il ne s'agit pas de crédibiliser les idées du concurrent mais de montrer qu'on sait être conciliant dans l'interaction, afin de construire de soi-même une image de personne courtoise). Dans le débat qui nous intéresse au contraire, l'objectif est de défendre la légitimité du programme commun, au-delà de défendre sa propre candidature. La relation entre les deux candidats fait référence à une histoire politique partagée et à un texte commun (le programme du PS) qui sous-tendent l'ensemble du discours. On entend donc, au travers de l'échange, ces discours qui sont perceptibles dialogiquement dans la manifestation des formes d'accord.

\section{Manifester son désaccord}

On traitera dans ce second point les différentes marques de dissensus, c'est-à-dire les éléments qui révèlent l'opposition au cœur du débat politique télévisé. En effet, les deux candidats participent à ce débat pour se différencier et pour montrer aux téléspectateurs que chacun est meilleur que l'autre. 
Toutefois, dans la conduite globale du débat, il faut noter que les candidats utilisent fréquemment des stratégies d'évitement du désaccord. Alors que les animateurs tentent de mettre en relief les divergences entre les deux et les critiques sous-entendues par le discours d'un des deux adversaires, ces derniers nient souvent ces accusations et se contentent de présenter leurs propres idées sans entrer dans la polémique (cf. les prises de parole 72 et 88 dans l'exemple 11, ci-dessous). Le candidat recentre alors son discours sur son propre programme en faisant abstraction de ce que l'autre a pu dire, sous-entendre ou penser. Ainsi, la défense de son projet ne passe pas nécessairement par l'attaque du projet adverse. On trouve néanmoins certaines marques de désaccord, les critiques entre les deux peuvent ainsi être plus ou moins explicites. Comme précédemment, on traitera les différents cas selon un axe progressif: d'abord les cas où le discours de l'autre est simplement amendé, puis ceux où le discours de l'autre est mis à distance, enfin les cas où il est proprement disqualifié.

\subsection{Amender le discours de l'autre}

Cette première catégorie présente les cas où le désaccord reste tempéré. Il ne s'agit pas de s'opposer frontalement au discours de l'autre, mais plutôt de se l'approprier et de lui apporter des modifications. On traitera successivement des cas où un candidat reformule les termes de l'autre et ceux, plus marqués, où il apporte des rectifications.

\subsubsection{Reformulation}

Le cas de reformulation est un type de reprise discursive spécifique : le contenu est sensiblement identique, mais le lexique employé est différent et l'appréciation même de l'objet du discours varie selon le locuteur. L'exemple 10 présente une reformulation très particulière: $\mathrm{FH}$ renchérit sur le discours de MA et accentue sa portée ${ }^{13}$. En 35, DP demande à MA si elle s'engage à faire campagne pour FH s'il est désigné candidat du parti socialiste. Celle-ci introduit sa réponse par l'énoncé « c'est évident » (36). Il pose ensuite la même question à $\mathrm{FH}$ :

41 DP lundi si martine aubry l'emporte vous serez à cent pour cent derrière elle

$42 \quad$ FH c'est une évidence et une exigence $\downarrow+$ l'évidence c'est que ces primaires ont été organisées pour c'que ce rassemblement pourra être et c'est une exigence parce qu'il ne peut pas y avoir de victoire s'il n'y a pas d'unité $\downarrow$

En 42, il y a une continuité thématique : FH reprend les propos de MA avec l'expression «c'est une évidence » et renchérit avec «et une exigence ». La conjonction de coordination «et» permet de poursuivre sur l'accord préalable de l'évidence en question, mais il est complété par la partie droite du « et ». FH marque ainsi un accord de départ, c'est l'initiale même de son propos, mais le marque en deux dimensions. L'accord initial n'est donc qu'un accord partiel à partir duquel un désaccord ultérieur est possible (dans la poursuite du renchérissement) ou perceptible par cet ajout complémentaire. C'est un effet d'annonce qui se développe dans ce même tour. Au passage, il est intéressant de noter que la reprise n'est pas à l'identique du point de vue de la catégorie grammaticale. FH effectue une nominalisation de l'adjectif « évident » proposé par MA. Ce changement marque une essentialisation du propos. Comme le notent Garric et Léglise, à propos du discours patronal, ici aussi ce glissement « témoigne d'un discours plus dépersonnalisant qu'impersonnel. Le procès semble se déployer de luimême, sans aucun contrôle agentif ou comme le résultat d'une instance autre, probablement la conjoncture » (2003: 125). Ceci permet de déposséder personnellement MA de cet argument pour l'essentialiser et en faire une valeur partagée (ce qui va aussi dans le sens du rassemblement des 
candidats socialistes). Il constitue un point de départ comme point d'accord qui permet de mettre davantage en valeur le renchérissement sous forme de complément avec la notion d'exigence.

L'exemple suivant présente un cas de reformulation qui se poursuit sur plusieurs tours de parole et qui révèle une guerre des dénominations au travers des dialectiques identitaires. Dans les constructions des identités discursives, le concept de dialectique du Même et de l'Autre (Détrie, $2001: 81-82^{14}$ ) permet de montrer comment l'image de soi se crée par jeu d'opposition. Ici l'incarnation du candidat se réalise dans le substantif « gauche » et c'est dans sa qualification que le débat va être lancé. Le journaliste DP va d'abord questionner MA sur une attaque personnelle qu'elle aurait pu adresser indirectement à son adversaire socialiste en définissant leur différence entre une "gauche dure » qu'elle symboliserait et une «gauche molle » dont FH serait le représentant ${ }^{15}$. DP fait ici référence à la sortie de MA lors du troisième débat des primaires socialistes en date du 5 octobre 2011, lorsqu'elle précise que sa « conviction profonde c'est qu'on ne pourra pas battre une droite dure [...] si on est une gauche molle ». L'attaque rhétorique n'identifie pas nommément $\mathrm{FH}$, mais elle est tournée de sorte à ce que l'interdiscours permette au plus grand nombre d'identifier sa cible.

68 MA j'ai dit et je le redis que face à une droite dure face à une crise qui dure il faut une gauche effectivement qui ne soit pas molle une gauche forte $h[\ldots]$

$$
[\ldots]
$$

71 DP mais qui est cette gauche molle $\uparrow$

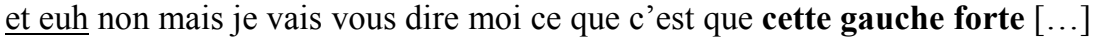
DP et vous ne visiez personne

74 MA j'ai dit ce que j'étais + et moi j'vais dire quand j'ai qu'j'étais je défendrais une gauche forte personne ne m'a posé la question de savoir si j'étais forte ou pas j'ai pas besoin de m'expliquer ou d'argumenter pour dire que je ne suis pas une gauche molle $\downarrow+$ ou une gauche faible $\downarrow$ vous vous êtes senti visé françois hollande + et blessé même non et d'ailleurs je constate que:: martine aubry euh n'évoquait personne donc j'en suis très heureux $\downarrow+$ il aurait pu y avoir méprise $\uparrow+$ malentendu c'n'est pas l'cas $\downarrow$ moi je n'sais pas ce que c'est que la gauche dure je n'ai pas envie d'une gauche dure $\downarrow$ très franchement on sort de cinq ans d'une présidence brutale et on serait nous une candidature sectaire $\uparrow$ je ne le veux pas $\downarrow$ mmh (mouvement de la tête)

$78 \quad$ FH je pense que le pays a besoin d'être apaisé réconcilié $\downarrow+$ je suis pas non plus une gauche molle on n'a pas besoin là d'une gauche TImide il faut une gauche qui soit SOLide parce que ya effectivement un choc c'est la crise il faut être capable euh de la rel'ver cette crise [...] [donc il faut être solide et puis il va falloir avoir euh une grande cause j'ai évoqué l'école et l'éducation et en même temps il faut une gauche sincère parce que si elle n'est pas sincère si elle n'est pas crédible si elle ne fait que des promesses qui n'peuvent pas être tenues] [...] $[\ldots]$

85 DP et quand vous dites sectaire [vous pensez à martine aubry $\uparrow$ ]

$88 \quad$ FH \& non $\uparrow$ je ne s- je n'ai pas pris ces concepts là je ne suis ni dans la gauche molle ni dans la gauche dure je suis dans la gauche solide et sincère $\downarrow$

89 DP bien alors la crise vous l'évoquiez martine aubry

90 MA non attendez moi je veux dire un mot quand même euh:: oui la gauche dure encore qu'être dur avec les banques aujourd'hui être du avec certains privilégiés ça sera pas mal mais je pense que dans l'état de notre pays + il faut le rassembler il faut rouvrir le champ de la négociation + il faut s'ouvrir vers les français c'est 
ce que j'ai voulu avec ces primaires donc c'est un peu un symbole de la façon dont je souhaite diriger le pays le présider euh simplement quand je parle de gauche forte la force c'est une bonne chose c'est comme la solidité ça veut dire aussi qu'il faut dire les choses clairement [...]

Néanmoins, il y a, dans ce passage, la confrontation de deux paradigmes. Le premier présenté, celui de MA se définit sur une opposition gauche «dure» (ou «forte») et gauche «molle» selon DP. Le second paradigme s'axe sur la solidité et la sincérité. Le premier, à la différence du second, se détermine par une dialectique très marquée. L'antonymie «dure/molle » participe de cet effet de sens. La référence à la mollesse est sans doute à voir en parallèle avec l'opposition RPR de 1995 qui avait mis en scène les candidats Jacques Chirac et Édouard Balladur pour l'élection présidentielle. Avant l'élection, ce dernier avait l'avantage des sondages (comme FH dans le cas présent) mais faisait l'objet de critique sur sa «mollesse » ou en tous cas de railleries notamment par des humoristes (tels que les Guignols de l'info comme le montre Coulomb-Gully, 1999: 209). Dire que MA tente un tel rapprochement mériterait néanmoins une analyse bien plus détaillée qui dépasserait le cadre de ce débat afin d'observer le dialogisme de la nomination (tel Siblot, 2001). Néanmoins, dans cet échange télévisé, chaque fois MA ne répond pas à la question de DP, elle fait des «enchaînements thématiques problématiques » (Sandré, 2010), plus précisément un « détournement de thème » (ibid.) restant sur sa définition de « la gauche forte », sans expliciter « la gauche molle ». C'est par opposition à la Droite, puis au gouvernement et enfin particulièrement aux banques que MA justifie l'emploi du vocable « forte ». Elle prétend que c'est une qualité qui lui revient en allant de soi (74). Un raisonnement fallacieux par syllogisme pourrait alors laisser entendre que FH serait donc ce fameux «mou». À l'inverse, FH opère par dénégation et refuse de reconnaître l'opérativité, voire la pertinence d'une dureté dans un tel combat politique. Il prône la solidité et la sincérité. L'argumentaire de la solidité rejoint celui de la force que développe MA. En revanche celui de la sincérité renvoie au « durcissement» du ton et de la campagne de son adversaire. Lui est plus connu pour ses blagues que pour ses phrases assassines, s'appuie sur un long passé de premier secrétaire du PS. Il peut alors appuyer la longévité de cet exercice sur l'argument de la sincérité par respect de ses propres attitudes politiques.

Ces cas de reformulations permettent à chaque candidat d'exposer ses propres mots et ses propres définitions. Si les candidats ne sont pas clairement en désaccord (quel candidat de la gauche ne voudrait pas d'une gauche forte ou solide?), ils se présentent toutefois chacun comme les maîtres de leurs propres idées, comme le montrent ces négociations lexicales.

\subsubsection{Rectification}

Les cas de rectification sont un pas supplémentaire dans le désaccord, il ne s'agit plus de reformuler le discours de l'autre pour l'adapter à ses propres mots, mais de le corriger. C'est donc un amendement plus catégorique. Nous n'avons relevé qu'un exemple, où MA vient amender le discours de FH :

125 MA $[\ldots]$ sur le cumul des mandats $\uparrow$ chacun sait euh ce qu'il y a à en penser $\downarrow+$ en tout cas moi je suis HEUreuse de l'avoir défendu y compris parfois contre mes intérêts parce que je crois que les français sauront que la VRAIE rénovation du parti socialiste c'est d'faire rentrer des jeunes des femmes h heu + des hommes et des femmes de toutes les cultures et ils sauront qui l'a fait $\downarrow$

127 FH ça s'ra fait après deux mille douze $\downarrow$

129 MA oui ça s'ra fait dès maintenant parce que là on va choisir les députés pour deux mille $\underline{\text { douze } \downarrow}$ 
130 FH et et non non ça s'ra fait a- a[près deux mille douze $\downarrow$ ]

132 MA ah non non non on et bé pour moi c'est très clair je l'ai fait voter par les militants $\downarrow$

133 FH si ça s'ra fait applica- ça s'ra applicable en deux mille douze pour le parti socialiste $\downarrow$

134 MA voilà $\downarrow$ y compris pour les députés qu'ils choisiront dans quelques jours $\downarrow$

En 127, FH vient apporter une précision en prolongeant le discours de MA. En 129, celle-ci corrige l'indication temporelle qu'il a donnée. S'ensuit un passage avec de nombreux chevauchements où les deux candidats - d'accord sur le fond - s'opposent sur la date d'application de la réforme sur le cumul des mandats. La rectification apportée par la candidate correspond au rôle qu'elle se donne dans la mise en place de cette réforme : en 125, elle se positionne clairement comme l'instigatrice du projet en tant que première secrétaire du parti socialiste. Elle doit donc montrer qu'elle en maîtrise parfaitement le calendrier.

Amender le discours de l'autre permet d'introduire des divergences, tout en se basant sur un certain consensus. Il s'agit chaque fois d'apporter de menues distinctions, soit simplement lexicales pour le cas des reformulations, soit sur le contenu pour le cas des rectifications, tout en affichant un accord plus global.

\subsection{S'opposer au discours de l'autre}

Dans les exemples suivants, les candidats s'opposent plus clairement au discours de l'autre. Cependant, la formulation du désaccord reste ponctuelle et assez limitée. Comme dans le point 1.1., on trouve des phénomènes exprimant un désaccord minimal, et d'autres montrant simplement ce désaccord, sous forme de répétition niée.

\subsubsection{Désaccord minimal}

De même que les adverbes confirmatifs servaient à signaler un accord, les adverbes négatifs peuvent servir à indiquer un désaccord. Cependant on trouve peu d'occurrences de ce type dans le débat. L'exemple 13 montre le seul cas relevé :

(13)

195 FH [le contrat d'génération il ne coûte rien $\downarrow+++$ donc vous pos'rez] la question $\uparrow \backslash$

$197 \quad$ FN il est financé c'est ++ huit milliards d'euros $\uparrow$

198a FH oui mais il est il est pris sur les exonérations d'cotisations sociales existantes bon $\downarrow$ donc euh c'est une \&

199 MA ah oui mais c'est

198 b FH \& mesure c'est \&

200 MA ah non

198c FH \& c'est une mesure que j'vais l'expliquer [hein puisque vous m'interrogez là-dessus]

Dans cet extrait, MA est l'auteur de deux prises de paroles en chevauchement. La première est interrompue, la seconde est achevée. Si elle semble concéder un accord en 199, elle introduit immédiatement une opposition avec le mais. Lorsqu'elle reprend la parole en 200, la forme d'accord a disparu au profit de l'adverbe négatif. Cependant ici, elle ne poursuit pas son propos ni ne l'argumente. Elle indique seulement à son interlocuteur - et aux téléspectateurs - qu'elle n'est pas d'accord avec la solution proposée par FH (à laquelle elle s'opposera plus frontalement par la suite, cf. les exemples 17, 18 et 19). Dans cet exemple, l'adverbe négatif correspond à la manifestation minimale du désaccord : MA se positionne simplement en opposition avec le discours tenu par FH. 


\subsubsection{Désaccord montré}

Le désaccord peut être montré sous forme de reprise textuelle niée. L'opposition au discours de l'autre est alors plus marquée et peut être argumentée. L'occurrence relevée concerne une critique de MA, qui qualifie les contrats de génération proposés par $\mathrm{FH}$ de "nouvelle niche fiscale » et d' «effets d'aubaine » (221). Ces deux appellations sont reprises par DP lorsqu'il donne la parole à FH pour répondre ${ }^{16}$. Ces deux éléments lexicaux sont donc mis en évidence par l'animateur, il est donc normal que le candidat les reprenne à son tour pour se positionner vis-à-vis d'eux. C'est ce qu'il fait au tour 226 :

(14)

226 FH alors première réponse euh d'abord il y aura aucun effet d'aubaine puisque les entreprises ont déjà ces exonérations d'cotisations sociales elles les garderont donc il n'y aura aucune perte de ce point de vue de compétitivité [...]

FH fait donc ici référence à l'attaque de MA, et y répond en niant le syntagme «effet d'aubaine ». Il justifie ensuite son avis en argumentant sa proposition. Ce cas de répétition niée est le seul cas où la reprise est montrée, ici ni par le locuteur ni par l'interlocuteur (comme dans les cas traités en 1.1.2.) mais par l'animateur. C'est bien lui qui permet la formulation de ce désaccord en ayant extrait des interventions de MA les deux critiques principales. Il y a sans doute d'autres cas où un candidat reprend à l'autre ses mots pour les nier, mais alors la reprise n'est marquée par aucun participant au débat. Nous n'avons retenu ici que le cas où le désaccord est montré par un des locuteurs engagés dans l'échange.

Le désaccord peut apparaitre ponctuellement dans le débat sans faire l'objet d'une argumentation : c'est le cas de l'emploi de l'adverbe négatif utilisé seul et de la reprise lexicale niée, mis en évidence par un des participants au débat.

\subsection{Disqualifier le discours de l'autre}

Dans ce troisième sous-point le désaccord prend une forme plus polémique : il s'agit de disqualifier le discours de l'autre. Ce sont des phénomènes qui n'apparaissent que ponctuellement dans le débat sur des thèmes précis. Les trois exemples relevés sont le fait de MA qui critique ouvertement les idées de son concurrent ${ }^{17}$. Il est intéressant de noter que ces trois extraits se situent dans la première demi-heure du débat.

Le premier cas concerne la question du retour à l'équilibre budgétaire de la France. Après avoir interrogé FH (qui prône le retour à l'équilibre en 2017), DP donne la parole à MA sur ce même thème : celle-ci répond que sur l'échéance en 2017 elle est «réservée parce qu'[elle] pense que ce n'est pas possible de l'annoncer [...] même si [...] c'est un objectif bien évidemment» (167). Elle critique donc ouvertement le discours de $\mathrm{FH}$, qu'elle accuse de faire des effets d'annonce sans être sûr de pouvoir tenir ses promesses. La disqualification du projet de son adversaire tient dans sa probable infaisabilité, soulignée ici par MA. Elle se positionne, elle, comme une candidate plus réaliste, prenant en compte l'ensemble de la situation ( « le climat actuel [...] la situation internationale et la croissance française », 167). La disqualification de l'adversaire est donc intimement liée à une auto-valorisation.

Le second cas concerne la question de la règle d'or - proposée par le président de la République Nicolas Sarkozy à l'été 2011. C'est MA qui introduit elle-même ce thème (sans qu'il soit demandé par un animateur) pour mieux marquer la différence entre les deux candidats :

(15)

90 MA [...] il faut qu'on dise clairement les choses tu parles à juste titre du fait qu'il faut bien gérer la france + et ça c'est pas moi qui dirai le contraire $\downarrow$ mais par exemple sur la règle 


\begin{abstract}
d'or que nous avait proposée nicolas sarkozy + cet été toi tu as dit oui et je vais la reprendre peut-être même dès le la loi d'finances et en tout cas si je suis élu moi j'ai dit euh moi j'veux pas d'une règle où on m'dit qu'demain on sera sérieux je propose tout de suite au président de faire un certain nombre de choses qui permettent à la fois de réduire les déficits publics mais aussi de relancer la croissance et l'emploi parce que ce sont les problèmes des français $\downarrow$
\end{abstract}

Elle introduit donc ce thème en exposant la réaction de chacun des candidats : celle de $\mathrm{FH}$ se trouve du côté de l'actuel président de la République (i.e. leur adversaire à tous les deux), celle de MA se situe dans l'opposition. Il est intéressant de noter qu'elle commence son attaque par une marque appuyée d'accord : elle valide (sous la forme «tu parles à juste titre ») le discours de FH « il faut bien gérer la France ». Elle introduit donc d'abord un consensus global sur la tâche qui incombe à un président de la République, puis poursuit immédiatement par un exemple qui les oppose frontalement. FH répond à cette attaque en mettant à distance la proposition de Nicolas Sarkozy «c'est pas moi qui ai inventé ce concept » (96) et son application : le président voulait l'inscrire dans la constitution, il lui répond «ça n'aura pas besoin d'une modification de la constitution euh la loi y suffira » (100). Il prend donc ses distances avec Nicolas Sarkozy, et se place ainsi lui-même dans l'opposition. Mais MA n'est pas convaincue :

(16)

$100 \quad$ FH $\quad[\ldots]$ puis-je être plus clair $\downarrow$

101 MA oui je pense $\downarrow+$ mais euh surtout sur la règle d'or bon voilà tu as quand même au

départ dit parce que c'était un moment où il fallait donner l'impression qu'on était plus sérieux qu'les sérieux euh qu'il fallait prendre cette règle d'or

102 FH non jamais [jamais + tu ne trouveras jamais aucun écrit sur la règle d'or de ma part]

109 MA je les ai hein mais j'vais on va pas

110 FH ben vas-y donne-les-moi $\downarrow$

111 MA on va pas les les envoyer en perm- on va pas s'les envoyer en permanence mais

112 FH et et:: ।

113 MA voilà nan mais tu as dit d'abord je vais l'inscrire dans la loi d'finances et puis \

114 FH ah mais c'est pas pareil $\uparrow$ la loi d'finance c'est pas [du tout la règle d'or c'était l'fait de réduire les déficits $\downarrow$ ]

119 MA tu as dit je vais inscrire la règle d'or dans la loi d'finances puis après tu as dit

120 FH c'est pas la règle d'or c'est la réduction du déficit $\downarrow$

121 MA d'accord alors moi j'ai donné en tout cas une règle claire [...]

Ce passage présente de nombreuses occurrences marquant le désaccord entre les deux candidats. La critique de MA est basée sur l'emploi du discours rapporté : elle impute à FH un discours direct (comme elle le faisait déjà en 90) chaque fois introduit par le verbe dire (101,113, 119), et certifié par des preuves - qu'elle ne dévoilera pas - $(109,111)$. L'attaque menée ici est donc bien en règle et préparée par la candidate, ce qui explique qu'elle introduit cette question d'elle-même. La référence au discours de l'autre (tenu antérieurement au débat) est bien ici une stratégie de disqualification de la position de son adversaire. Elle s'applique également ici à discréditer sa façon de se défendre : si elle clôture ce passage par l'adjectif claire (qu'elle s'attribue), elle commence par l'accuser (en 101) de n'être pas assez clair. Pourtant la question posée par FH en 100 joue plutôt le rôle d'une question rhétorique : il affirme ne pas pouvoir être plus clair. MA prend alors la parole pour lui répondre et se positionner immédiatement dans une position très critique vis-à-vis de lui.

Le troisième cas, enfin, concerne les contrats de génération, proposés par FH (dont nous avons déjà parlé pour l'exemple 13). MA critique d'abord le financement et le coût de cette proposition : 
(17)

223 MA [...] nan mais le prix huit milliards quand françois dit on va les prendre sur les exos d'charge moi j'préfère lier les exos d'charges + euh euh à l'égalité des salaires homme-femme $[\ldots]$

$[\ldots]$

226a FH $[\ldots]$ les entreprises ont déjà ces exonérations d'cotisations sociales elles les garderont donc il n'y aura aucune perte de ce point de vue de compétitivité ou d'alourdissement du compte \&

227 MA mais ca coute huit milliards $\uparrow$

226b FH \& elles auront de toute façon ces exonérations de cotisations sauf que y aura un senior qui sera resté un peu plus longtemps $\downarrow[\ldots]$

En 223, il s'agit, encore une fois, pour MA, de disqualifier le discours de l'autre sous forme de discours rapporté direct. Elle enchaîne aussitôt sur ses propres propositions : elle oppose ainsi leurs deux projets et se positionne, elle, comme la défenseur de «l'égalité des salaires homme-femme ». En 226a, alors que FH défend son idée, MA intervient en chevauchement pour insister sur le coût de sa proposition qui impliquera selon elle des sacrifices sur d'autres points. Cet énoncé vise simplement à marteler cet argument (déjà cité auparavant comme en 223) pour écorcher l'image de bon gestionnaire de son interlocuteur: elle cherche ainsi à décrédibiliser sa candidature, et l'accuse de vouloir dépenser à mauvais escient. Cet argument est ensuite repris en 234 :

234 MA et pour faire un tutorat moi je suis totalement favorable aux tuteurs mais les tuteurs on n'a pas besoin de payer les entreprises pour qu'ils fassent toi-même tu étais chez $p$ $\mathrm{s} \mathrm{a}^{18}$ et tu leur as dit vous faites exactement ce que je veux faire + mais tu les as pas payé pour ça $\downarrow[\ldots]$

Comme dans l'exemple 15, elle introduit sa critique par la validation du discours de l'autre « je suis totalement favorable aux tuteurs »: elle est donc d'accord sur le principe, mais non sur l'application proposée par FH. Elle disqualifie ensuite la proposition de FH en mettant en scène son concurrent lors d'une visite d'entreprise et en rapportant ses propos (toujours sous forme de discours direct) : elle conclut cet épisode par l'évidence de la non-utilité du financement du projet de FH. MA revient ainsi à plusieurs reprises sur cette question pour critiquer ouvertement son adversaire, en utilisant différentes formes d'atténuation : outre la forme de validation - très claire - de son discours, elle utilise sans cesse le discours de $\mathrm{FH}$ pour le confronter à ses propres dires (les choses semblent ainsi se démontrer d'ellesmêmes). Pour autant, la disqualification est très claire. Il faut noter que MA revient sur cette question, vers la fin du débat, et est alors plus radicale :

603 MA [...] voyez on parlait tout à l'heure dans le fond du contrat génération et je le dis en toute amitié à françois ça ne marche pas $\downarrow+$ et c'est là où l'expérience a un sens + parce qu'on l'a fait $\downarrow$ on sait qu'ça marche pas $\downarrow+$ j'écoute les syndicats + je sais qu'ils ont raison je crois RÉellement que l'expérience est nécessaire $\downarrow[\ldots]$

Si la prise de parole débute par le témoignage de son amitié, elle poursuit immédiatement par un avertissement: son projet n'est pas opérationnel. Cette disqualification de la proposition de son adversaire lui permet de valoriser sa propre candidature, dont la pertinence est basée sur l'expérience (dont elle accuse FH de manquer). Cette dernière critique sur le contrat de génération est sans appel : comme il s'agit de la fin du débat, elle n'utilise plus les mêmes procédés pour argumenter ou défendre son point de vue. Ce dernier est acté par les syndicats (qui sont appelés ici pour jouer le rôle d'adjuvant de la première secrétaire du parti socialiste) et n'est pas présenté comme étant discutable comme 
précédemment. La fin du débat radicalise donc les positions. Il faut toutefois noter que si la critique est explicitement destinée à $\mathrm{FH}$, ce dernier a, dans le discours de MA, le statut de délocuté ${ }^{19}$ : elle ne s'adresse pas directement à lui, et ne le regarde pas. En ce sens, elle évite la confrontation directe.

Les différents cas de disqualification du discours de l'autre - tous du fait de MA - sont utilisés par la candidate pour valoriser sa propre candidature aux yeux des téléspectateurs. Il s'agit pourtant de moments très circonscrits dans le débat, portant sur des thèmes précis, développés au tout début du débat. Néanmoins sa détermination ne disparaît pas à la fin de l'interaction, comme le montre l'exemple 19 , où MA reprend ses propos antérieurs pour une dernière pique à son adversaire.

Les phénomènes discursifs témoignant un désaccord entre les candidats sont moins nombreux que ceux témoignant un accord. Les cas où l'opposition est clairement exprimée et argumentée sont assez rares. Le dissensus apparaît surtout sur des sujets précis, disséminés dans un consensus plus global. Il faut par ailleurs noter que certains témoignages de désaccord sont introduits par une forme de consensus, il s'agit alors d'atténuer l'offense et de ménager la face de son interlocuteur. Ces manifestations de désaccord sont caractéristiques du genre débat et de la confrontation politique lors d'un face à face entre deux tours d'une élection. De plus, il faut noter la référence à la campagne interne au parti - notamment aux discours tenus lors des trois débats précédant le premier tour ${ }^{20}$ - et aux clivages qu'elle a développés. Toutefois, les points de divergences ne concernent que quelques points très particuliers et il ne s'agit pas d'une opposition globale: les deux adversaires partagent bien les mêmes principes politiques.

\section{Bilan}

Dans ce débat de l'entre-deux tours des primaires socialistes, on retrouve des caractéristiques propres au débat politique télévisé en période d'élection et des caractéristiques propres à un échange entre collaborateurs politiques. L'analyse s'est ici focalisée sur les marques plus ou moins explicites du consensus et du dissensus. Ce dernier est bien présent et la confrontation entre les deux candidats est réelle. Cependant, les deux candidats s'opposent surtout sur des points précis de leur programme respectifs et sont plutôt d'accord sur les principes. Il y a bien un socle commun à partir duquel chacun développe ses propres solutions. Le débat est donc gouverné par un consensus global. Or si les interactions relevant du genre débat politique télévisée présentent généralement quelques phénomènes discursifs à visée collaborative, il apparaît clairement que dans ce corpus-ci, les phénomènes témoignant l'accord sont bien plus présents que dans un débat politique classique - a fortiori un débat de l'entre-deux tours (Sandré, 2010). Plus que de simples témoignages de consensus, il faut noter une véritable collaboration entre les deux candidats et la défense commune d'une certaine vision de la politique et d'un idéal de gauche. L'ennemi officiel n'est pas celui qui est en face mais clairement celui qui sera en face lors du débat de l'entre-deux tours de l'élection présidentielle de 2012. Nicolas Sarkozy (qui est présenté comme cet adversaire, bien que sa candidature ne soit pas encore déclarée) est d'ailleurs cité à plusieurs reprises dans le débat, pour être systématiquement mis à distance.

L'analyse des différentes occurrences montre un déséquilibre entre les deux candidats : MA témoigne plus fréquemment son accord et son désaccord avec le discours de FH que l'inverse. Sur les 29 occurrences mentionnées, MA est l'auteur de 22 phénomènes discursifs. On peut donc affirmer que la candidate dialogue davantage avec le discours de son adversaire que ne le fait $\mathrm{FH}$. Celle-là utilise fréquemment les arguments et les exemples utilisés par celui-ci, elle cite à plusieurs reprises des paroles tenues par FH dans le débat ou précédemment; et ce, indifféremment pour témoigner son soutien et pour émettre des critiques. Il est par ailleurs notable que FH ne s'oppose jamais frontalement à son 
interlocutrice: il n'est l'auteur d'aucun cas de disqualification du discours de l'autre relevé dans le corpus, et le seul cas de désaccord montré est en fait une réponse à l'animateur qui insiste sur les critiques de MA. Il est donc plutôt dans une position de fuite du conflit, contrairement à elle. Cette répartition des rôles a ainsi contribué à forger une image de MA très belliqueuse face à un FH plus posé : le fait d'être favori dans les sondages lui permet d'imposer sa candidature, sans avoir besoin de se battre pour cela.

\section{Références bibliographiques}

Bres J. et al. (éds) (2005). Dialogisme, polyphonie : approches linguistiques. Bruxelles : Be Boeck.

Calbris G. (1979). L’Index signifiant. Études de linguistique appliquée, 35, 91-109.

Charaudeau P. (2005). Le discours politique. Les masques du pouvoir. Paris : Vuibert.

Constantin de Chanay H. (2010). Adresses adroites, les FNA dans le débat Royal-Sarkozy du 02 mai 2007. In Kerbrat-Orecchioni C. (éd.), S'adresser à autrui. Les Formes nominales d'adresse, Chambéry: Éditions de l’Université de Savoie, 249-294.

Coulomb-Gully M. (1999). Rhétorique télévisuelle et l'incarnation politique. Éléments de réflexion. Réseaux, 94, 195-213.

Détrie C., Siblot P., Verine B. (éds) (2001). Termes et concepts pour l'analyse du discours. Paris: Honoré Champion.

Dufour F. et Rosier L. (à paraître). Héritages et reconfigurations conceptuelles de l'analyse du discours «à la française » : perte ou profit ? Langage et société, 140.

Garric I. et Léglise I. (2003). Quelques caractéristiques du discours patronal français. Mots, 72, 113-133.

de Gaulmyn M.-M. (1987). Reformulations et planification métadiscursive. In Cosnier J. et Kerbrat-Orecchioni C. (éds), Décrire la conversation, Lyon : Presses Universitaires de Lyon, 203-223.

Guilbert T. (2011). L'"évidence » du discours néolibéral. Analyse dans la presse écrite. Broissieux : Éditions du croquant.

Hailon F., Richard A. et Sandré M. (éds) (à paraître). Le Discours politique identitaire. Le Discours et la langue, 5.

Jeanneret T. (1999). La Co-énonciation en français. Berne : Peter Lang.

Kerbrat-Orecchioni C. (1990). Les Interactions verbales. Paris : Armand Colin.

Rémi-Giraud S. et Constantin de Chanay H (2007). Démocratie et ses dérivés. De la dénomination à l'argument sans réplique? Mots, 83, 81-99.

Richard A. (2007). Black ou Noir, emprunt d'identité ou identité d'emprunt. Neologica, 1, 145-161.

Richard A. et Sandré M. (soumis). Accords et désaccords : construction de points de vue dans un débat télévisé sur le cinéma. Actes du Colloque Représentations du sens linguistique $V$.

Sacks H., Schegloff E.A. et Jefferson G. (1974). A Simplest Systematics for the Organization of Turn-Taking for Conversation. Language, 50, 4, 696-735.

Sandré M. (à paraître). Discours rapporté et stratégies argumentatives : Royal et Sarkozy lors du débat de l'entredeux tours. Langages et société, 140.

Sandré M. (2011a). Dialogisme, comportement et débat politique télévisé : Ségolène Royal lors du débat de l'entredeux tours. In Jaubert A. et al. (dir.), Citations II. Citer pour quoi faire? Pragmatique de la citation, LouvainLa-Neuve : Harmattant-Academia, 239-256. 
Sandré M. (2011b). Quand le dialogisme détourne le dialogue : un cas d'“enchaînement non pertinent". Actes du Colloque international Dialogisme: langue, discours, URL: http://recherche.univmontp3.fr/praxiling/spip.php?article264.

Sandré M. (2010). Constantes et spécificités des dysfonctionnements interactionnels dans le genre débat politique télévisé : une application au débat de l'entre-deux tours de l'élection présidentielle de 2007. Thèse de doctorat, Université Montpellier 3

Siblot P. (2001). De la dénomination à la nomination. Les dynamiques de la signifiance nominale et le propre du nom. Cahiers de Praxématique, 36, 189-214.

Verine B. et Détrie C. (éds) (2011) L'Actualisation de l'intersubjectivité : de la langue au discours. Limoges : Lambert-Lucas.

${ }^{1}$ Un débat politique télévisé : le débat de l'entre-deux tours de l'élection présidentielle de 2007 (Sandré, 2010, 2011a et (à paraître)) et un débat culturel télévisé : l'émission de cinéma Le Cercle (Richard et Sandré, (soumis)).

${ }^{2}$ Voir notamment Kerbrat-Orecchini, $1990: 18-24$.

${ }^{3}$ On a exclu de l'analyse toutes les manifestations de ce type de la part des animateurs : les régulateurs émis par ces derniers répondant à d'autres visées que ceux émis entre les débatteurs.

${ }^{4}$ Conventions de transcription, mises en évidence ici par les guillemets français : "et on en saura rien" chevauchement de parole ; «\&» continuation du tour de parole après un chevauchement; «\» interruption

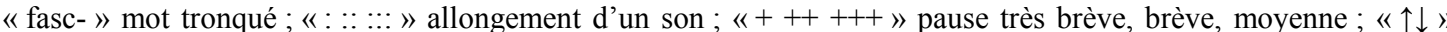
intonation montante/descendante; «> ..<» accélération du débit de parole; «BELLE»: accentuation de la syllabe; "(hochement de la tête)» : indication non verbale. Certains extraits ont été modifiés pour une meilleure lisibilité ce qu'indiquent les crochets, c'est pourquoi il peut manquer des numéros dans la colonne de gauche. Le gras signale le phénomène analysé.

${ }^{5}$ Nous avons relevé une seule occurrence d'accord montré sous forme de discours rapporté, mais il s'agit en fait d'un faux accord: MA interpelle FH ainsi «puisque tu m'parlais tout à l'heure de droite dure » (221), mais c'est elle qui a utilisé cette expression en 68 (cf. l'exemple 11). Elle veut ainsi indiquer qu'elle reprend et approuve les mots de son adversaire, mais le consensus est faussé par cette référence à son propre discours.

${ }^{6} \mathrm{CSG}$ : Contribution Sociale Généralisée.

${ }^{7}$ On retrouve également ce geste chez SR dans le débat face à Nicolas Sarkozy (Sandré, 2011a : 245-246).

${ }^{8}$ On retrouve également la configuration inverse : une réponse négative après l'annonce d'une divergence par l'animateur (MA 272)

${ }^{9}$ RATP : Régie Autonome des Transports Parisiens.

${ }^{10}$ C'est le cas en 264a et 265 : FH présente sa réforme de la fiscalité française qui «se fera sur l'année deux mille douze ", MA intervient en chevauchement pour confirmer cette date.

${ }^{11}$ On trouve également ce cas en 511 : alors que FH dit «j'ai pu observer que les états-unis viennent voter au congrès viennent de voter au congrès une clause de sauvegarde » (510), MA intervient en chevauchement pour affirmer : «si ils viennent de décider des clauses de sauvegardes au contraire ». Les propos ne sont pas exactement identiques, mais les deux disent pourtant bien la même chose, et MA, encore une fois, soutient très clairement son adversaire.

${ }^{12} \mathrm{Au}$ sens de Jeanneret (1999) : ce phénomène renvoie à une construction collaborative et continue d'un énoncé par deux locuteurs distincts, l'achèvement de l'énoncé étant assuré par une autre personne que celle qui l'a initié.

${ }^{13}$ On retrouve ce même procédé en 385 : MA renchérit sur la question du retour de l'âge légal de la retraite à soixante ans. Elle reprend cette idée à $\mathrm{FH}$, mais introduit immédiatement un amendement : «non seulement je suis 
d'accord mais je vais plus loin ». Si elle valide explicitement le discours de son concurrent, elle se positionne plus en avant sur cette question et montre ainsi qu'elle est plus déterminée que lui.

${ }^{14}$ Termes et concepts pour l'analyse du discours, Détrie, Siblot, Verine (éds.), entrée « Dialectique langagière ».

15 « est-ce qu'il y a ce soir face à face deux gauches $\downarrow$ martine aubry vous avez dit il y a une gauche dure ou une gauche forte + et puis il y a une gauche molle $\downarrow$ est-ce que vous parliez du projet politique de françois hollande ou d'françois hollande lui-même » (DP 67).

${ }^{16}$ «bon alors une nouvelle niche un effet d'aubaine un effet d'aubaine françois hollande $\downarrow$ » (222).

${ }^{17}$ Il lui arrive même de se positionner dans le rôle de l'intervieweur (ou de l'institutrice) « je voudrais juste vérifier pour aller au bout des choses parce que les français attendent qu'on soit précis » (444) avant de lui demander de préciser le chiffrage de sa proposition de recruter soixante mille fonctionnaires (pour mieux s'y opposer).

${ }^{18}$ PSA : Peugeot Société Anonyme (groupe Peugeot Citroën).

${ }^{19}$ Sur la délocution in prasentia, cf. Constantin de Chanay, $2010: 288$

${ }^{20}$ Le 15 septembre 2011, le 28 septembre 2011 et le 05 octobre 2011, entre les six candidats : FH, MA, Arnaud Montebourg, Manuel Valls, Ségolène Royal et Jean-Michel Baylet. 